\title{
ALGORITMO EVOLUTIVO PARA A ESTIMAÇÃO DA FREQUÊNCIA EM SISTEMAS ELÉTRICOS UTILIZANDO FPGAS
}

\author{
Denis V. Coury* \\ couryesc.usp.br \\ Mário Oleskovicz* \\ oleskesc.usp.br \\ Daniel Barbosa $a^{\ddagger}$ \\ daniel.barbosaepro.unifacs.br \\ Alexandre C. B. Delbem \\ acbdeicmc.usp.br \\ Eduardo V. Simões ${ }^{\dagger}$ \\ simoes@icmc.usp.br \\ Janison R. de Carvalho* \\ janisonesc.usp.br \\ * Depto de Engenharia Elétrica- Escola de Engenharia de São Carlos -EESC \\ Universidade de São Paulo - USP \\ Av. Trabalhador Sancarlense, 400 Centro \\ CEP 13566-590 - São Carlos - SP \\ †'Instituto de Ciências Matemáticas e de Computação -ICMC \\ ${ }_{\ddagger}^{\ddagger}$ Universidade Salvador, Mestrado em Energia, Rua Ponciano de Oliveira, 126, 2ž andar, Rio Vermelho, 41.950-275, \\ Salvador, BA
}

\begin{abstract}
Evolutionary Algorithm for Frequency Estimation in Electrical Systems Using Fpgas

This paper presents an efficient technique for frequency estimation in Electrical Power Systems (EPS) based on Genetic Algorithms (GAs). Frequency estimation of a distorted electrical signal is modeled as an optimization problem. The motivation concerning the use of the GA approach is related to its intrinsic robustness to noise. The GA is implemented in a FPGA (Field-Programmable Gate Array) device and the estimation process is computed in real-time. This is made possible due to (a) the implicit parallelism of FPGAs (b) the suitable choice of the GA operators to explore this parallelism and (c) the determination of the appropriate architecture for a FPGA that parallelizes the GA routine and ensures its good performance. In order to evaluate the performance of the

Artigo submetido em 18/02/2010 (Id.: 01103)

Revisado em 01/05/2010, 18/11/2010, 22/03/2011

Aceito sob recomendação do Editor Associado Prof. Antonio Carlos Zambroni de Souza
\end{abstract}

proposed method, an EPS was simulated using the ATP software for some typical operation conditions. The resulting signals were analyzed by the proposed GA/FPGA approach and they were compared to the performance of a commercial relay. Promising results were found with the proposed methodology.

KEYWORDS: Frequency Estimation, Genetic Algorithms, Field-Programmable Gate Arrays, Digital Relaying, Power System Protection.

\section{RESUMO}

Este trabalho apresenta uma técnica eficiente para a estimação da frequência em Sistemas Elétricos de Potência (SEPs) baseada em Algoritmos Genéticos (AGs). A estimação da frequência de um sinal elétrico é modelada como um problema de otimização. A motivação do uso de AGs nesta abordagem é a sua intrínseca robustez ao ruído presente nos sinais. O AG proposto é implementado em um dispositivo FPGA (Field-Programmable Gate Array) e 
o procedimento de estimação da frequência é realizado em tempo real. Isto é possível devido: (a) ao paralelismo implícito dos FPGAs no processamento das instruções, (b) a escolha de operadores apropriados dos AGs para explorar este paralelismo e (c) a determinação da arquitetura do circuito para FPGA que paraleliza o AG, assegurando um desempenho satisfatório. Para a avaliação do desempenho da metodologia proposta, um SEP com condições de operação típicas foi modelado através do software ATP (Alternative Transients Program). Os sinais gerados deste sistema foram processados pela abordagem AG/FPGA proposta, sendo as respostas decorrentes da mesma comparada às respostas de um relé comercial. Resultados bastante promissores foram alcançados com a metodologia proposta.

PALAVRAS-CHAVE: Estimação da Frequência, Algoritmos Genéticos, Field-Programmable Gate Arrays, Relés Digitais, Proteção de Sistemas Elétricos de Potência.

\section{INTRODUÇÃO}

A estimação em tempo real da frequência em Sistemas Elétricos de Potência (SEPs) é uma tarefa extremamente importante em muitos campos da Engenharia Elétrica. Relés digitais de frequência utilizam esta estimação para tomar decisões importantes, como proteger os elementos do SEP contra perda de sincronismo quando sujeitos a sub ou sobrefrequência. Uma precisa estimação da frequência é também essencial para manter a estabilidade do SEP. Neste contexto, o equilíbrio dinâmico entre geração e carga, tem se tornado mais difícil devido à grande expansão dos sistemas elétricos. O crescente interesse pela Qualidade da Energia Elétrica (QEE) tem também estimulado os pesquisadores na busca de novas ferramentas e metodologias para uma estimação da frequência instantânea e precisa do sistema.

Várias pesquisas tem se direcionado para diferentes técnicas aplicadas a estimação da frequência. Phadke et al. (1983) e Wang e Sun (2004) apresentaram algoritmos baseados na aplicação da Transformada Discreta de Fourier (TDF). Dash et al. (1999) e Routray et al. (2002) apresentaram métodos de estimação da frequência complexa baseados na abordagem que utiliza o filtro de Kalman. Terzija et al. (1994), Ziarani e Conrad (2004) e Karimi et al. (2004) estudaram métodos que modelam esta estimação como problemas de otimização. Terzija et al. (1994) utilizam o algoritmo de Newton para resolução do problema apresentado. O método do gradiente descendente é utilizado por Ziarani e Conrad (2004) e Karimi et al. (2004), cujas equações não lineares resultantes são chamadas EPLL (Enhanced Phase-Locked Loop). Sachdev e Giray (1985) utilizaram o método de mínimos quadrados para a determinação da frequência de um SEP. Um algoritmo utilizando filtros adaptativos, também baseado na técnica de mínimos quadrados, foi apresentado por Barbosa (2007).
Neste caso, a análise do sistema de potência é realizada através da conversão das tensões trifásicas em um sinal complexo pela aplicação da transformada $\alpha \beta$, sendo estas direcionadas ao algoritmo de filtragem adaptativa.

Técnicas inteligentes também têm sido utilizadas para estimação da frequência em um SEP. Dash et al. (1997) apresentam uma abordagem baseada em Redes Neurais Artificiais. El-Naggar e Youssef (2000), Souza et al. (2008) e Coury et al. (2009) utilizaram os Algoritmos Genéticos (AGs) como ferramentas principais para a resolução do problema de otimização considerado.

Este artigo apresenta um método eficiente baseado em AGs para a estimação da frequência em um SEP. Uma janela móvel de dados, contendo amostras do sinal de entrada, é utilizada para se ajustar a uma onda senoidal pura. Dentre as principais contribuições do artigo, deve ser destacado:

- Implementação da proposta de um relé de frequiência utilizando um algoritmo genético em FPGA. Devido às propriedades intrínsecas do algoritmo genético (como a utilização de população, gerações, etc.) somada a sua aplicação em tempo real na proteção, respeitando a rápida velocidade de atuação requerida, a sua implementação em hardware é certamente um desafio. No entanto, com a capacidade de processamento em paralelo dos FPGAs e todo o seu projeto de implementação mencionado, essa realização prática foi possível com desempenho altamente satisfatório. A referida aplicação não seria possível com processadores convencionais. A principal contribuição deste artigo, comparado com trabalhos anteriores (Souza et al. (2008) e Coury et al. (2009)) é o desenvolvimento de um protótipo de relé de frequência para uma aplicação em tempo real. Esta experiência foi possível pela otimização das operações executadas pelo AG, visando à sua implementação em hardware, dispondo de FPGA, como será posteriormente descrito.

- Uma análise de custos associada à proposta, mostrando que um FPGA de baixo custo pode ser utilizado para a implementação descrita.

- Uma análise estatística dos resultados, mostrando a confiabilidade das estimativas do AG, mesmo com a variação da inicialização do processo.

$\mathrm{O}$ artigo apresenta a seguinte estrutura: a abordagem AG/FPGA proposta é apresentada na seção 2; o SEP simulado através do software ATP (Alternative Transients Program) (EEUG, 1987) para obtenção das formas de onda para análise está descrito na seção 3; a seção 4 apresenta os casos simulados, bem como os resultados da estimação 
da frequência quando comparados aos resultados de um relé comercial de frequência; e, finalmente, a seção 5 apresenta as conclusões finais do trabalho até então realizado.

\section{O AG/FPGA PROPOSTO}

\subsection{Algoritmos Genéticos}

Um AG é um algoritmo de busca baseado na genética e na teoria da evolução das espécies. Da genética, utilizam-se: (i) a representação de um indivíduo (uma solução para um problema) basicamente por seu cromossomo (array com valores para cada variável do problema) e (ii) os operadores de reprodução que manipulam os cromossomos para gerar novos indivíduos. Da teoria da evolução, utiliza-se o princípio de que a partir dos melhores indivíduos em uma determinada população, geram-se os descendentes formando uma nova população. Pode-se dizer que um AG opera sobre uma população de aproximações (potenciais soluções), que são inicialmente geradas de forma aleatória. A cada nova iteração (chamada geração), produz-se uma nova população, que tende a possuir soluções melhores, caminhando em direção ao ótimo do problema.

Cada cromossomo é avaliado através de uma função de avaliação (fitness) que verifica a sua representação a uma possível solução do problema analisado. Desta forma, quanto melhor for a representação do cromossomo, maior será sua chance de ser selecionado para reproduzir e passar suas características para os descendentes da próxima população. As técnicas utilizadas para selecionar indivíduos são chamadas de operadores de seleção, e as técnicas para gerar novos indivíduos são os operadores de reprodução.

Os operadores de reprodução mais utilizados são a roleta, o torneio e o truncamento (De Jong, 2006). Pela roleta, os indivíduos têm uma probabilidade de serem selecionados que é proporcional à razão entre o seu fitness e o fitness médio da população. Pelo torneio, seleciona-se o melhor (chamado de vencedor do torneio) de dois ou mais indivíduos escolhidos aleatoriamente da população.

Os operadores de reprodução são em geral o cruzamento e a mutação. O cruzamento troca informações genéticas (trechos de array com valores de variáveis) entre os indivíduos selecionados de modo a produzir novos indivíduos. O operador de mutação pode alterar, com certa probabilidade, os valores (chamados de alelos) de variáveis (chamadas genes) de um cromossomo dentro de uma faixa de variação. Em geral, aplica-se cruzamento sobre dois indivíduos, gerando dois novos indivíduos. Sobre estes, aplica-se então a mutação. Com isso, obtém-se uma parte da nova população. A outra parte é gerada aplicando-se somente a mutação sobre os indivíduos selecionados.
Na prática, um AG é utilizado para encontrar o máximo ou o mínimo da função objetivo de um problema. A função que calcula o fitness de um indivíduo pode ser a própria função objetivo, ou uma transformação linear, ou não-linear sobre esta função. Adicionalmente, o algoritmo de cálculo desta função deve ser rápido, uma vez que o mesmo irá avaliar cada indivíduo de uma dada população em suas sucessivas gerações.

Uma estrutura geral de um AG é mostrada abaixo, na qual $t$ é o índice da geração e $P(t)$ é a população na geração $t$.

$t \leftarrow 0$;

- Inicialize $P(t)$ aleatoriamente

- Avalie $P(t)$

- enquanto (critério de parada é falso)

- Aplique seleção a $P(t)$ para gerar $P_{A U X}$

- Aplique cruzamento e mutação em $P_{A U X}$

$-P(t+1) \leftarrow \mathrm{P}_{A U X}$

- Avalie $P(t+1)$

$-t \leftarrow t+1$

Três importantes características dos AGs são enfatizadas a seguir. Primeiramente, esses algoritmos podem determinar o valor ótimo de funções objetivas complexas, mesmo sendo estas discretas ou com suas derivadas não definidas. Adicionalmente, a inicialização randômica insere indivíduos na população em um espaço de busca global, definidos por valores máximos e mínimos de cada parâmetro da função objetivo, melhorando, geralmente, a busca pelo ótimo global em detrimento de ótimos locais. Por fim, o paralelismo implícito dos AGs permite gerar uma nova população com processamento paralelo, uma vez que a geração de um novo indivíduo depende somente de indivíduos provenientes de uma população prévia.

\subsection{O Problema de Otimização e o AG Proposto}

O problema de otimização usando AGs para estimação da frequência de um SEP é definido considerando o modelo senoidal do sinal de tensão, cuja análise é feita por uma janela móvel de dados com comprimento de um ciclo da frequência fundamental do sinal, conforme apresentado na Figura 1. Matematicamente, o valor da função custo no instante de tempo $n$ é definido como: 


$$
\begin{aligned}
& e[n]=C(n, A, f, \theta)= \\
& \quad \sum_{k=0}^{N-1}|u[n-k]-A \sin (2 \pi f k T+\theta)|
\end{aligned}
$$

Onde $N$ é o número de amostras da janela, $u$ é o sin de entrada e $\{A, f, \theta\}$ é a série de parâmetros amplitud frequiência e ângulo de fase, respectivamente, a ser estimar para minimizar a somatória, ou seja, para se aproximar c sinal de entrada.

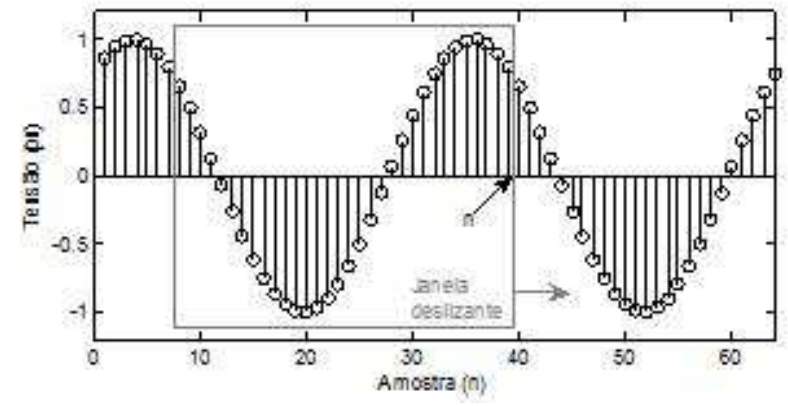

Figura 1: Janela móvel de dados sob análise no tempo $n$.

O processo de busca de uma solução ótima para minimizar a equação (1) é exemplificado na Figura 2. Neste exemplo, uma estação de trabalho processou o AG usando uma janela de um ciclo de um sinal elétrico com amplitude, frequência e fase de 1,0 pu, $60 \mathrm{~Hz}$ e 4,1888 rad., respectivamente. A Figura 2(a) mostra a população randômica inicial e, depois de 20 gerações, o processo de convergência do algoritmo pode ser observado na Figura 2(b). O melhor indivíduo de cada população é indicado pelas flechas, sendo que também pode ser observada a aproximação dos parâmetros aos valores reais à medida que as gerações são realizadas.

\subsubsection{Codificação}

Um esquema de código binário é usado para representar os parâmetros a serem estimados $\{A, f, \theta\}$. Cada indivíduo tem três sequências de bits correspondendo a três números inteiros, como mostrado na Figura 3. Deve-se observar que esses números inteiros são índices para posições em arrays de números reais, que armazenam os possíveis valores de cada variável com certo nível de discretização (quantização).

Assumindo um sinal de entrada normalizado e um limite de $\pm 2 \mathrm{~Hz}$ para a variação da frequência, o espaço de busca é mostrado na Tabela 1, bem como o número de bits para representar cada parâmetro.
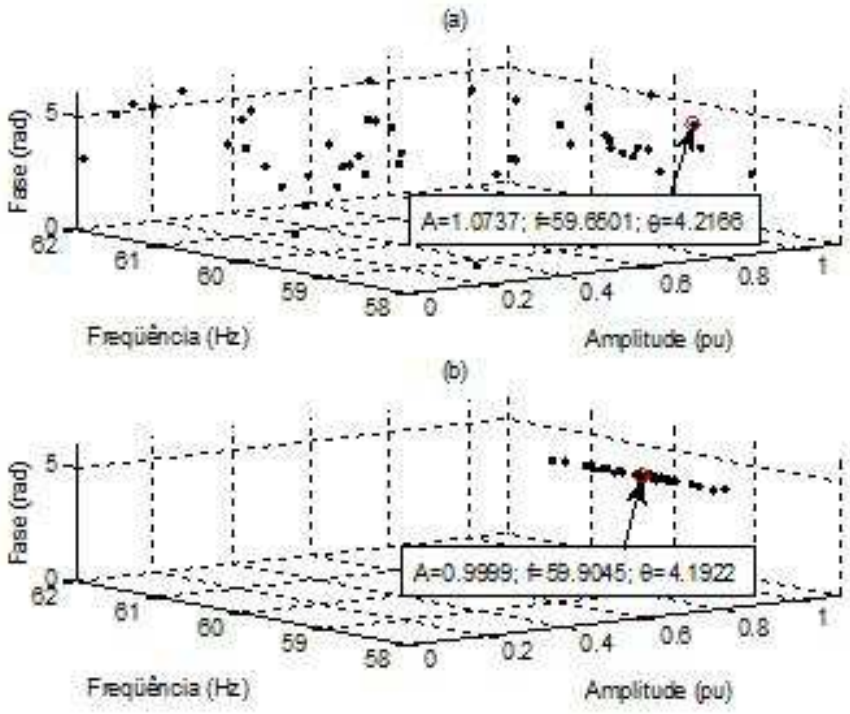

Figura 2: População de um AG para a (a) primeira e (b) vigésima geração.

Tabela 1: CODIFICAÇÃO DOS PARÂMETROS (ESPAÇO DE BUSCA)

\begin{tabular}{l|l|l|l}
\hline Parâmetro & $\begin{array}{l}\text { Número de } \\
\text { Bits }\end{array}$ & Faixa & Unidade \\
\hline$A$ & 8 & {$[0,75 ; 1,0)$} & $\mathrm{pu}$ \\
\hline$F$ & 24 & {$[58,0 ; 62,0)$} & $\mathrm{Hz}$ \\
\hline$\theta$ & 12 & {$[0,0 ; 2 \pi)$} & $\mathrm{Rad}$ \\
\hline
\end{tabular}

\subsubsection{Seleção}

O processo de seleção é o procedimento que possibilita que as soluções geradas pelo AG evoluam a cada geração. Basicamente, esse operador escolhe probabilisticamente os indivíduos que irão reproduzir. A seleção por torneio é utilizada neste artigo. Este procedimento tem apresentado melhores resultados que outros operadores de seleção para uma grande gama de aplicações (Goldberg (2002), Deb (2002) e De Jong (2006)). A seleção de dois candidatos para a reprodução (pais) é feita de acordo com o algoritmo abaixo. Quatro indivíduos $\{a, b, c, d\}$ são randomicamente escolhidos da população atual. Os pais $p_{1}$ e $p_{2}$ são resultados da competição entre os pares $\{a, b\}$ e $\{c, d\}$, respectivamente.

- seleção randômica de quatro indivíduos $\{a, b, c, d\}$;

- $\mathrm{p}_{1} \leftarrow$ melhor entre $\{\mathrm{a}, \mathrm{b}\}$ (comparando sua aptidão)

$$
\text { - } p_{2} \leftarrow \text { melhor entre }\{c, d\} \text { (comparando sua aptidão) }
$$

\subsubsection{Cruzamento}

O processo de cruzamento combina características de duas soluções (chamadas pais) possibilitando gerar soluções melhores que os pais. Mesmo que na vizinhança desses 


\begin{tabular}{|c|c|c|} 
Amplitude & Frequência & Fase \\
\hline$\underbrace{A_{N A-1} A_{N A-2} A_{N A-3} \ldots A_{0}}_{N_{A} \text { bits }}$ & $\underbrace{f_{N f-1} f_{N f-2} f_{N f-3} \ldots f_{0}}_{N_{f} \text { bits }}$ & $\underbrace{\theta_{N \theta-1} \theta_{N \theta-2} \theta_{N \theta-3} \ldots \theta_{0}}_{N_{\theta} \text { bits }}$ \\
\hline
\end{tabular}

Figura 3: Representação do indivíduo.

pais não haja soluções melhores, eles podem não ser a solução ótima ou não estar próximos dela. Porém, pelo cruzamento, por combinar características de ambos os pais, pode-se encontrar uma solução melhor. Com isso, o processo evolutivo avança e evita que o algoritmo fique preso a esses pais, que são ótimos locais.

$\mathrm{O}$ cruzamento entre dois pais, $p_{1}$ e $p_{2}$, está representado genericamente na Figura 4 , na qual $\psi=\{A, f, \theta\}$. Os parâmetros $\psi_{p 1}$ e $\psi_{p 2}$ são combinados e geram três novos valores possíveis (como mostrado na figura) a partir do cálculo do valor médio entre $\psi_{p 1}$ e $\psi_{p 2}$ e a distância $\delta$ entre eles. Os parâmetros escolhidos para os descendentes são determinados randomicamente dos cinco valores possíveis apresentados. Esse procedimento leva a $20 \%$ de probabilidade para escolha de cada valor, sendo realizado três vezes (uma vez para cada $\psi$ ).

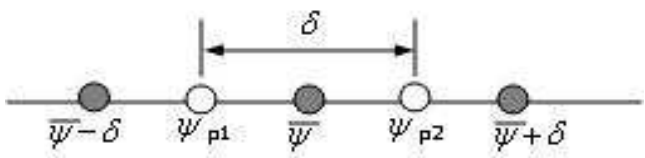

Figura 4: O operador de cruzamento.

\subsubsection{Mutação}

A mutação é o operador de reprodução responsável pela geração de diversidade na população. Em outras palavras, a mutação pode gerar valores de variáveis que nenhum indivíduo da população atual possui. Basicamente, esse operador é aplicado separadamente para cada gene do cromossomo. A mutação ocorre de acordo com certa probabilidade (denominada taxa de mutação) sobre cada variável representada em um cromossomo.

Neste trabalho, a mutação corresponde a adicionar ou subtrair o valor $1 \mathrm{em}$ um dos genes do cromossomo. Isso corresponde a pegar o próximo valor ou o anterior no array de valores discretizados daquela variável (ver Seção 2.2.1).

\subsection{Detalhes da Implementação do FPGA}

Pela teoria de AGs supracitada, verifica-se que um grande número de operações matemáticas é necessário, o que poderia criar uma barreira intransponível para a aplicação destes em sistemas em tempo real. Por exemplo, considere um sistema monitorado com um intervalo amostral de 1 ms, por um dispositivo FPGA, operando a $25 \mathrm{MHz}$. Ou seja, 20.000 pulsos do clock estariam disponíveis para a obtenção da solução ótima, o que implica que todos os passos do algoritmo genético devem ser realizados antes do aparecimento da próxima amostra. Assim, conclui-se que um processamento sequencial puro não é suficiente para resolver este problema. Desta forma, a capacidade de processamento paralelo intrínseco dos FPGAs permite a implementação dos AGs em hardware, possibilitando sua aplicação em sistemas de medição em tempo real.

É importante mencionar que, mesmo sendo os FPGAs apropriados para a implementação de metodologias computacionalmente complexas, algumas estratégias devem ser adotadas para adaptar o algoritmo pretendido a certas limitações específicas do hardware. Mais especificamente, é altamente desejável que somente operações algébricas simples (adição e subtração) e operações lógicas (AND, OR e NOT) sejam executadas, além do deslocamento de bits para a esquerda ou direita em uma sequência binária. Essas operações são intrínsecas da arquitetura do hardware, executando apenas um pequeno número de pulsos do clock. Os tópicos a seguir abordam alguns aspectos de implementação do hardware para o sistema desenvolvido nesta pesquisa.

\subsubsection{As operações de multiplicação e a função seno}

As operações de multiplicação são indesejáveis e as mesmas podem ser reduzidas, uma vez que uma multiplicação por um fator 2 é equivalente ao deslocamento a esquerda de um bit em uma sequência binária, podendo otimizar estas operações. Por exemplo, o termo $2 \pi k f T$ com $k=6$ é rapidamente calculado deslocando o termo com $k=3$ um bit para a esquerda.

A função seno também demanda uma atenção especial na otimização do algoritmo, dado o esforço computacional envolvido. Uma solução seria considerar somente alguns termos na expansão em série desta função. No entanto, esta estratégia implica no aumento de multiplicações aproximadamente na proporção do quadrado do número de 
termos utilizados na série. Desta forma, a estratégia usada foi a utilização de uma tabela seno, gerada e configurada na memória interna do FPGA. O tempo computacional para avaliar a função de aptidão foi reduzido significativamente pelo cálculo a priori da função seno, quando comparado ao tempo de cálculo em uma estação de trabalho e o grande número de amostras (por exemplo, 1024 pontos) utilizado.

\subsubsection{Números randômicos}

Outro aspecto relevante no AG/FPGA proposto é o processo utilizado para geração de números aleatórios necessários ao mecanismo proposto. Estes números serviram de base para os processos de seleção, cruzamento e mutação dos AGs, como mostrado anteriormente. A literatura apresenta vários geradores de números aleatórios desenvolvidos para aplicações em FPGAs. A estratégia utilizada neste trabalho foi a construção de uma tabela com números aleatórios calculados off-line em uma estação de trabalho. Essa tabela deveria possuir números suficientes para um curso completo do AG proposto, o que significaria a necessidade de muito mais memória que a comumente disponível em dispositivos FPGAs. Assim, uma tabela circular com números aleatórios foi implementada para assegurar o suprimento de números aleatórios para qualquer número de gerações requerido.

\subsubsection{Gerando uma população}

A produção de $d$ indivíduos novos de uma população pode ser basicamente obtida gerando $p$ cópias do circuito designado a produzir um indivíduo novo. A paralelização da geração de novos indivíduos é relativamente fácil, já que cada indivíduo é uma sequência de bits e os blocos de circuitos para modificação de bits pelos operadores de cruzamento e mutação são relativamente pequenos.

Deve ser mencionado que operações relativas a cruzamento e mutação foram otimizadas do ponto de vista prático. $\mathrm{O}$ cruzamento foi realizado com algumas adições e deslocamento a esquerda para executar a divisão por $2 \mathrm{e}$ obter o valor médio representado na Figura 4. A mutação foi feita com algumas adições de acordo com a taxa de mutação. Ainda em termos da implementação em hardware, a seleção usando torneio é claramente mais vantajosa do que a roleta e, portanto, foi utilizada neste trabalho.

Adicionalmente, a otimização da memória do FPGA foi obtida usando-se somente duas populações, a atual e a próxima. As operações do AG foram calculadas de acordo com a seleção aleatória dos indivíduos (possíveis pais) da população atual. Somente os descendentes foram guardados para a próxima população. Esta população então se tornou a população atual. Este procedimento foi repetido até que o número máximo de gerações foi alcançado. Finalmente, um critério de elitismo foi adotado para acelerar o processo de convergência, considerando que o primeiro indivíduo da próxima população é sempre o melhor indivíduo da população atual.

\subsection{A Determinação de um FPGA Apropriado}

Um AG/FPGA eficiente para estimação da frequência requer um balanço entre qualidade da estimação e número de portas lógicas para suporte à paralelização dos operadores genéticos. Uma paralelização direta de todos os operadores, como usualmente implementado em software, necessitaria de um número de portas indisponível nos FPGAs. A viabilidade de um FPGA que satisfizesse os requisitos do projeto e que estivesse disponível no mercado foi então investigada. A Tabela 2 apresenta as características requeridas para o AG desenvolvido. A Tabela 3 apresenta as características dos FPGAs: F1, F2 e F3. O número de indivíduos criados em paralelo é $p$. Do ponto de vista do tempo de processamento, a solução ideal seria $p=30$, ou seja, todos os indivíduos da população seriam gerados em paralelo. No entanto, esta estratégia necessitaria de um FPGA com grande capacidade de processamento, inviabilizando a aplicação pelo elevado custo. Pela Tabela 2 podemos concluir que é possível a construção de um relé baseado em um dispositivo FPGA que seja rápido suficiente para a estimação da freqüência. Nestes termos, o FPGA F1 necessita menos de $0,2 \mathrm{~ms}$ para processar uma janela de dados (veja coluna para $p=2$ na Tabela 2 ) e apresenta um preço razoável dentre os analisados.

Tabela 2: Requisitos do AG e adequação do FPGA

\begin{tabular}{l|lll}
\hline Característica & \multicolumn{3}{|l}{ Necessidades do AG } \\
\hline $\begin{array}{l}\text { Número } \\
\text { de funções } \\
\text { combinatórias }\end{array}$ & 5.339 & $p=2$ & $p=4$ \\
\hline Bits de memória & 404.736 & 408.064 & 305.664 \\
\hline Registradores & 2.989 & 3.032 & 3.031 \\
\hline DSPs & 16 & 32 & 32 \\
\hline $\begin{array}{l}\text { Desempenho do } \\
\text { AG (ms)* }\end{array}$ & 0,355 & 0,177 & 0,089 \\
\hline $\begin{array}{l}\text { FPGA** } \\
\text { Escolhido }\end{array}$ & F1 & F1 & F2 \\
\hline Preço do FPGA & US\$995 & US\$995 & US\$ \\
\hline
\end{tabular}

*_ Milissegundos para produzir uma estimação de frequência adequada para uma janela de dados, onde adequado significa a percentagem de erro na estimação da frequência abaixo de 0,089 .

**_F2 é usado se F1 não pode suportar o paralelismo indicado por $\mathrm{p}$. 
Tabela 3: Características dos FPGAs F1, F2 e F3

\begin{tabular}{l|lll}
\hline FPGA & $\begin{array}{l}\text { F1 / ALTERA } \\
\text { ep2560f672c3 }\end{array}$ & $\begin{array}{l}\text { F2 / ALTERA } \\
\text { ep2s180f1508c5 }\end{array}$ & $\begin{array}{l}\text { F3 / ALTERA } \\
\text { ep4sg230kf40c2n }\end{array}$ \\
\hline $\begin{array}{l}\text { Elementos } \\
\text { Lógicos }\end{array}$ & $60 \mathrm{k}$ & $180 \mathrm{~K}$ & $680 \mathrm{k}$ \\
\hline Pinos & 493 & 1.171 & 1.517 \\
\hline $\begin{array}{l}\text { Bits de } \\
\text { memória }\end{array}$ & $2,5 \mathrm{M}$ & $9,3 \mathrm{M}$ & $22,4 \mathrm{M}$ \\
\hline DSP & $\begin{array}{l}288 \\
(9 x 9 \text { bits })\end{array}$ & $\begin{array}{l}768 \\
(9 \times 9 \text { bits })\end{array}$ & $\begin{array}{l}1,360 \\
(18 \times 18 \text { bits })\end{array}$ \\
\hline Preço & US\$995 & US\$ 1.995 & US\$ 4.495 \\
\hline
\end{tabular}

\section{O SISTEMA ELÉTRICO DE POTÊNCIA SIMULADO}

A Figura 5 mostra a representação do sistema elétrico utilizado nas simulações de manobras de cargas e faltas consideradas para avaliar o algoritmo de estimação da frequência proposto. $\mathrm{O}$ sistema elétrico é composto por um gerador síncrono de $13,8 \mathrm{kV}$ e potência aparente de 76 MVA, transformadores elevadores com relações de 13,8/138 $\mathrm{kV}$ e potência aparente de $25 \mathrm{MVA}$, linhas de transmissão com extensões variando entre 80 e $150 \mathrm{~km}$, transformadores abaixadores similares aos elevadores e cargas caracterizadas por um fator de potência de 0,92 indutivo e potência aparente variando entre 5 e $25 \mathrm{MVA}$.

As Tabelas 4 e 5 mostram os valores utilizados para caracterizar os diversos componentes via simulações computacionais dispondo do software ATP.

Na Tabela $4, S$ é a potência nominal, $N_{p}$ é o número de pólos, $V_{L}$ é a tensão nominal de linha, fé a frequência, $I F D$ é a corrente de campo, $R_{a}$ é a resistência de armadura, $X_{l}$ é a reatância de dispersão não saturada, $X_{o}$ é a reatância de sequência zero não saturada, $X_{d}$ é a reatância síncrona de eixo direto não saturada, $X_{q}$ é a reatância síncrona de eixo em quadratura não saturada, $X_{d}^{\prime}$ é a reatância transitória de eixo direto não saturada, $X_{d}^{\prime \prime}$ é a reatância subtransitória de eixo direto não saturada, $X_{q}^{\prime \prime}$ é a reatância subtransitória de eixo em quadratura não saturada, $\tau$ ' ${ }_{d o}$ é a constante de tempo transitória em vazio de eixo direto, $\tau$ " ${ }_{d o}$ é a constante de tempo subtransitória em vazio de eixo direto e $\tau{ }^{\prime \prime}{ }_{q o}$ é a constante de tempo subtransitória em vazio de eixo em quadratura.

É importante salientar que foi utilizado o modelo de linha de transmissão JMARTI vinculado ao software ATP. O modelo JMARTI provê uma tabela com os pólos e zeros da função de transferência ajustada para representar a variação da impedância característica da linha de transmissão em função da frequiência. Desta maneira, possibilita uma melhor representação do comportamento do sistema frente às perturbações provenientes de desequilíbrios entre geração e carga. A Figura 6 ilustra o comportamento da impedância

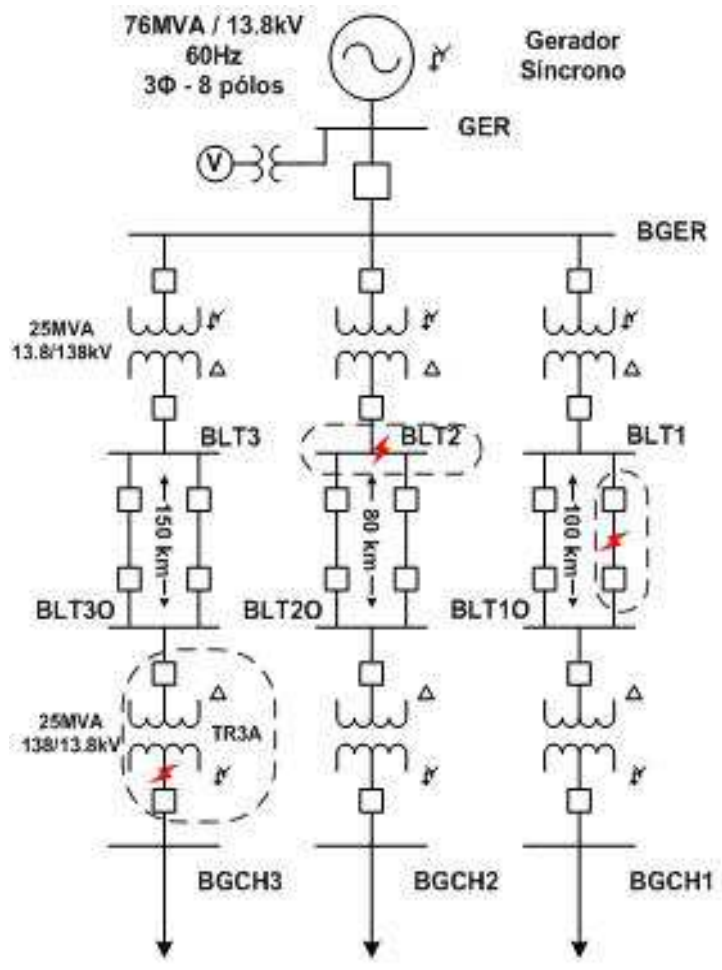

Figura 5: Representação do SEP analisado.

Tabela 4: Dados do gerador síncrono utilizado na simulação.

\begin{tabular}{llll}
\hline Descrição & Valor (un) & Descrição & Valor (un) \\
\hline$S$ & $76(\mathrm{MVA})$ & $N_{p}$ & 8 \\
$V_{L}$ & $13,8\left(\mathrm{kV}_{r m s}\right)$ & $f$ & $60(\mathrm{~Hz})$ \\
$I F D$ & $250(\mathrm{~A})$ & $R_{a}$ & 0,004 (p.u.) \\
$X_{l}$ & 0,175 (p.u.) & $X_{0}$ & 0,132 (p.u.) \\
$X_{d}$ & 1,150 (p.u.) & $X_{q}$ & 0,685 (p.u.) \\
$X_{d}^{\prime}$ & 0,310 (p.u.) & $X_{d}^{\prime \prime}$ & 0,210 (p.u.) \\
$X_{q}^{\prime \prime}$ & 0,182 (p.u.) & $\tau{ }^{\prime \prime}{ }_{d o}$ & 5,585 (s) \\
$\tau{ }^{\prime \prime}{ }_{d o}$ & 0,036 (seg.) & $\tau{ }^{\prime}{ }_{q o}$ & 0,073 (s) \\
\hline
\end{tabular}


da linha de transmissão com a variação de carga. Maiores informações sobre a silhueta e demais características da linha de transmissão podem ser encontradas em Barbosa (2007).

Tabela 5: Dados dos transformadores de potência.

\begin{tabular}{|c|c|c|}
\hline Transformadores de potência & $\mathbf{R}_{+}(\Omega)$ & $\mathbf{L}_{+}(\mathbf{m H}$ \\
\hline Imped. primária do transf. abaixador & 0,0175 & 1,514 \\
\hline $\begin{array}{l}\text { Imped. } \\
\text { abaixador }\end{array}$ & 1,048 & 90,83 \\
\hline Imped. primária do transf. elevador & 1,0 & 90,83 \\
\hline Imped. secundária do transf. elevador & 0,0175 & 1,514 \\
\hline
\end{tabular}

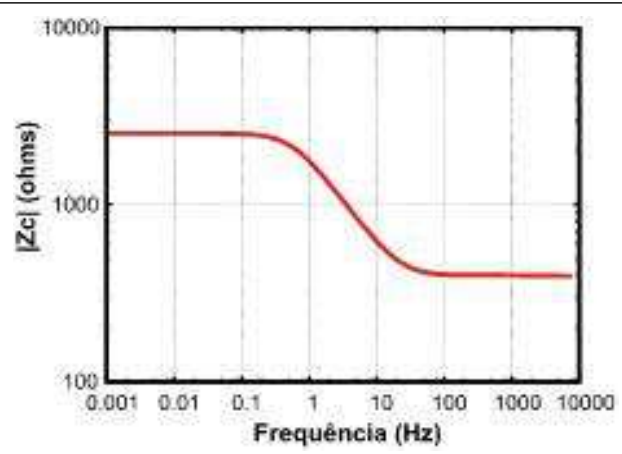

Figura 6: Comportamento da impedância da linha de transmissão em função da variação da carga.

Cabe ressaltar que o gerador síncrono foi caracterizado por uma conexão estrela aterrado, sendo o modelo utilizado via ATP, o de uma máquina dinâmica síncrona trifásica, já que este considera os diversos parâmetros elétricos e mecânicos do gerador. Além do gerador, foi aplicado o controle de velocidade dinâmico para sistemas hidráulicos (Vieira Filho, 1984) para permitir uma melhor análise dos resultados, bem como a regulação automática de tensão (AVR) (Lee (1992), Boldea (2006) e Mukherjee e Goshal (2007)). A equação 2 mostra a função de transferência do regulador de velocidade utilizado.

$$
\frac{\eta(s)}{\Delta F(s)}=-\frac{1}{R} \cdot \frac{1+s T_{r}}{\left(1+s T_{g}\right)\left(1+s \frac{r}{R} T_{r}\right)}
$$

Nesta, $\eta(s)$ é a posição do servo motor que aciona a turbina, $\Delta F(s)$ é o desvio de frequência, $R$ é o estatismo permanente, $r$ é o estatismo transitório, $T_{g}$ é a constante de tempo do regulador de velocidade e $T_{t}$ é a constante associada ao estatismo transitório. A tabela 6 apresenta os valores dos parâmetros utilizados no regulador de velocidade.

\section{RESULTADOS OBTIDOS COM A NOVA APLICAÇÃO}

Esta seção apresenta alguns resultados da metodologia proposta na pesquisa em questão. Quatro casos de operação anormal foram simulados para o sistema elétrico
Tabela 6: Parâmetros do regulador de velocidade.

\begin{tabular}{ll}
\hline Descrição & Valor (un) \\
\hline Constante de Tempo $\left(T_{g}\right)$ & $0,600(\mathrm{~s})$ \\
Constante de Tempo Dashpot $\left(T_{r}\right)$ & $0,838(\mathrm{~s})$ \\
Estatismo Transitório $(r)$ & 0,279 \\
Estatismo Permanente $(R)$ & 0,100 \\
Constante de Inércia do Gerador $(M)$ & $1,344(\mathrm{~s})$ \\
Constante de Tempo da Água $\left(T_{w}\right)$ & $0,150(\mathrm{~s})$ \\
\hline
\end{tabular}

apresentado na Figura 5. Cada condição aborda um comportamento dinâmico particular no balanço de potência e, consequentemente, na variação da frequência do sistema.

As medições do relé comercial (função 81) foram obtidas utilizando-se os sinais de tensão provenientes do programa ATP e aplicadas ao mesmo através de uma caixa de testes. As estimativas de frequência coletadas do equipamento são usadas na comparação dos resultados, nos quais a frequência real do sistema estudado foi medida diretamente da velocidade angular do gerador síncrono.

Com relação à metodologia proposta, o experimento foi feito conectando-se o FPGA a porta serial do microcomputador. Este por sua vez, envia uma amostra do sinal de tensão normalizado sob análise, com um intervalo de tempo de $1,3 \mathrm{~ms}$, simulando o dispositivo conectado a um sistema de potência real através de conversores $\mathrm{A} / \mathrm{D}$, transformadores de potencial e filtros analógicos (anti-aliasing e outros).

As simulações realizadas via o software ATP apresentam uma taxa amostral de $10 \mathrm{kHz}$, sendo que um filtro digital foi necessário para prevenção do efeito aliasing. Assim, um filtro Butterworth de segunda ordem, com frequência de corte de $200 \mathrm{~Hz}$, foi incluído na simulação antes da reamostragem com fator down-sample 13. Outro filtro Butterworth de segunda ordem, com frequência de corte de $5 \mathrm{~Hz}$, foi utilizado na saída do algoritmo para suavizar a estimação. O AG foi processado com uma janela de dados de 16 amostras, 30 indivíduos por população e com critério de parada de 300 gerações. O dispositivo FPGA utilizado foi um Altera Stratix II. Algumas das situações analisadas são apresentadas nos próximos tópicos.

\subsection{Uma Conexão Abrupta de Blocos de Carga}

Neste caso, alguns blocos de carga são conectados no barramento BCGH3 no tempo $t=2 \mathrm{~s}$. A frequência antes do chaveamento estava estabilizada no seu valor nominal de 60 Hz. Depois da inserção da carga, a frequência oscila como mostrada na Figura 7. A figura mostra a referência e os resultados da estrutura AG/FPGA utilizada, bem como de um relé comercial. As estimativas estão dentro de uma banda de erro de $0,2 \%$ e elas seguem a variação da frequência ocorrida 
no teste. O erro médio quadrático neste caso é de $3,75 \times 10^{-4}$ e $2,43 \times 10^{-4}$, para o método do AG/FPGA e para o relé comercial respectivamente.

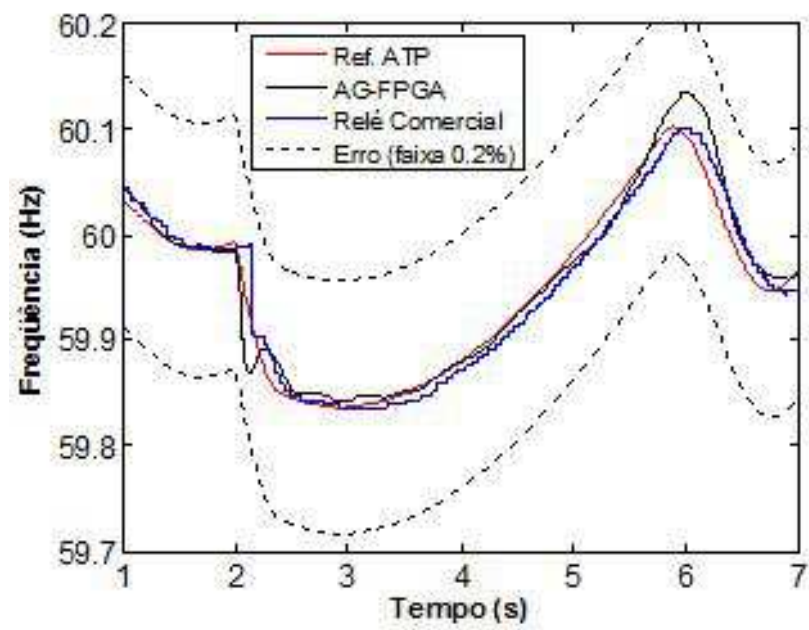

Figura 7: Estimação da frequência: o caso de uma conexão abrupta de um bloco da carga.

\subsection{Energização do Transformador TR1E}

Neste caso, o sinal analisado é obtido durante a energização do transformador TR1E, iniciado no tempo $t=1 \mathrm{~s}$. A frequência neste caso decresce como mostrada pela curva de referência do ATP da Figura 8. Adicionalmente, esta figura mostra os resultados para a abordagem AG/FPGA, bem como para o relé comercial. Ambas as estimativas seguem o decréscimo em frequência. $\mathrm{O}$ erro médio quadrático neste caso é $5,77 \times 10^{-3}$ e $1,87 \times 10^{-3}$, para o método do AG/FPGA e para o relé comercial respectivamente.

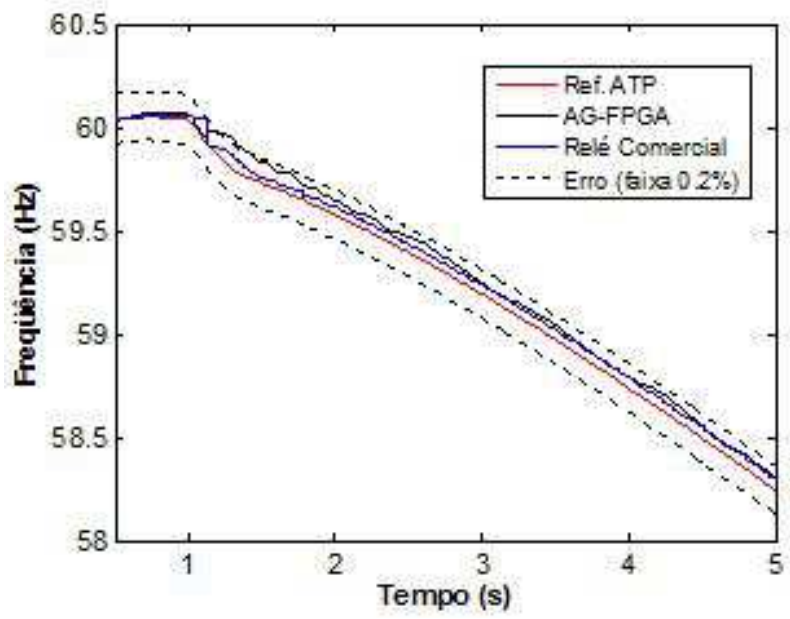

Figura 8: Estimação da frequência: o caso da energização do transformador TR1E.

\subsection{Uma Desconexão Abrupta dos Transformadores TR1E e TR3E}

Este caso analisa o impacto da saída dos transformadores TR1E e TR3E, ocorrida a 1,0 s. Pode ser observado, pela Figura 9, o aumento da frequência do sistema. A metodologia AG/FPGA e o relé comercial apresentam estimativas condizentes com esse aumento da frequência, não extrapolando a banda de erro de $0,2 \%$ e com erros médios quadrados de $2,56 \times 10^{-3}$ e $4,15 \times 10^{-3}$, para a estrutura proposta e o relé comercial, respectivamente.

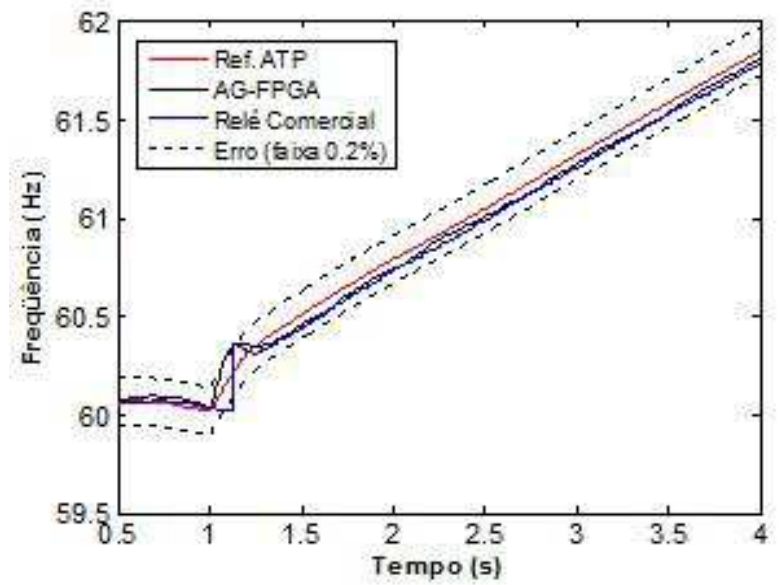

Figura 9: Estimação da frequência: o caso de uma desconexão abrupta dos transformadores TR1E e TR3E.

\subsection{Uma Falta Permanente a $50 \%$ da linha 1}

Neste caso, uma falta fase-terra (AT) foi simulada a 50 km do barramento BLT1 na linha de transmissão 1, a 2,5s. A frequência apresenta uma característica oscilatória que é amenizada no decorrer do tempo, como mostrado na Figura 10. O relé comercial apresenta erros significativos próximos à ocorrência do distúrbio, alcançando a estimação de $60,5 \mathrm{~Hz}$ quando o valor real é $60,2 \mathrm{~Hz}$. No entanto, o erro diminui e as estimativas permanecem dentro da banda de erros de $0,2 \%$, incluindo as estimativas do método proposto. Em geral, tanto o dispositivo AG/FPGA quanto o relé comercial apresentam estimativas adequadas da freqüência, sendo os erros médios quadráticos de $1,41 \times 10^{-3}$ e $3,67 \times 10^{-3}$, respectivamente.

\subsection{Análise Estatística da Resposta do AG-FPGA}

Esta seção apresenta o resultado do estudo estatístico das estimações de frequência obtidas pelo AG-FPGA após 30 execuções para cada um dos 10.000 pontos do sinal no caso de uma conexão abrupta de blocos de carga (item 4.1) com diferentes inicializações, conforme ilustra a Figura 11. 


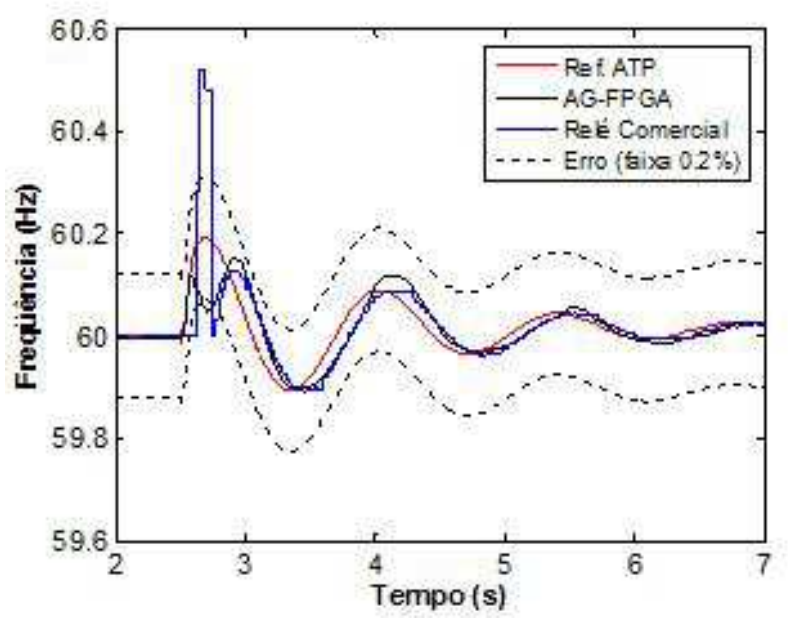

Figura 10: Estimação da frequência: o caso de uma falta permanente a $50 \%$ da linha 1 .

Nesta figura é possível observar as curvas com os valores máximo, mínimo e médio resultantes dessas estimações. Cabe observar que a diferença entre estes é desprezível, sendo percebida apenas por meio do destaque da figura supracitada. Tal afirmação é confirmada pelo desvio padrão de $7,2 \times 10^{-3}$ na pior das 300.000 estimações realizadas, comprovando que o resultado do algoritmo proposto é constante e independe da incialização dos indivíduos.

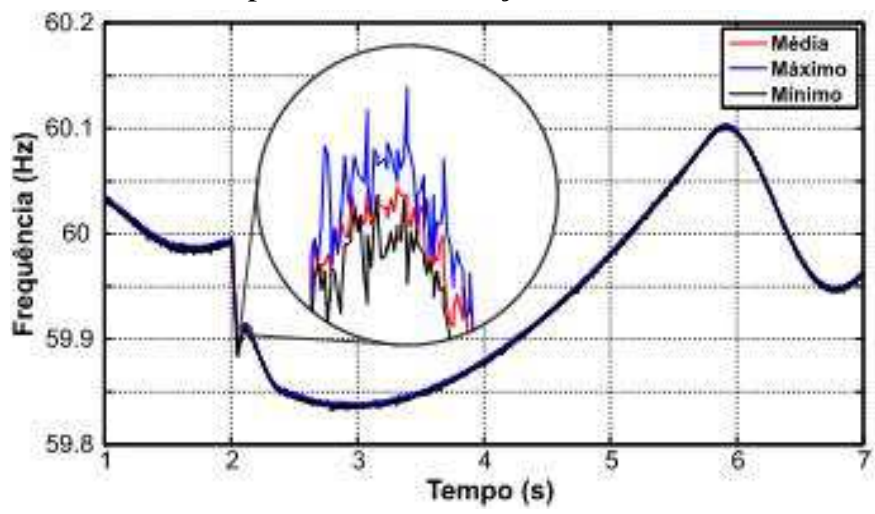

Figura 11: Estimação da frequência com diferentes inicializações.

\section{CONCLUSÃO}

Este trabalho apresentou uma implementação em hardware de AGs para estimação da frequência utilizando FPGAs. O algoritmo desenvolvido foi cuidadosamente estudado para otimização de todas as suas operações matemáticas a serem executadas na arquitetura em hardware. Um sistema elétrico de potência bastante completo foi simulado usando o software ATP para testar o esquema proposto. Embora a proposição tenha sido testada em inúmeras situações, por brevidade, quatro casos foram apresentados neste artigo. Em geral, pode ser afirmado que o desempenho do método proposto é superior ao relé comercial usado para comparação. No entanto, a principal contribuição desta proposta é o desenvolvimento de um dispositivo baseado em AG/FPGA para estimação da frequência a um custo bastante baixo. Adicionalmente, esta proposição demonstra que aplicações de AG/FPGA podem perfeitamente cumprir os requisitos de velocidade apresentados por um dispositivo de proteção em tempo real.

\section{AGRADECIMENTOS}

Os autores agradecem o apoio financeiro recebido por parte do $\mathrm{CNPq}$ (Conselho Nacional de Desenvolvimento Científico e Tecnológico), da CAPES (Coordenação de Aperfeiçoamento de Pessoal de Nível Superior) e da FAPESP (Fundação de Amparo à Pesquisa do Estado de São Paulo) em fases distintas de desenvolvimento deste trabalho. Os mesmos também agradecem ao Departamento de Engenharia Elétrica da Universidade de São Paulo Campus de São Carlos, por toda a infra-estrutura necessária para a elaboração deste trabalho.

\section{REFERÊNCIAS BIBLIOGRÁFICAS}

Barbosa, D. (2007) "Estimação da Frequência em Sistemas Elétricos de Potência por Filtragem Adaptativa", Dissertação de Mestrado. Escola de Engenharia de São Carlos. Universidade de São Paulo.

Boldea, I. (2006) Synchronous Generators. Boca Raton: CRC Press, ISBN: 084935725X.

Coury, D. V., Oleskovicz, M., Delbem, A. C. B., Simões, E. V., Silva, T. V., Barbosa, D. and Carvalho, J. R. (2009) "A Genetic Based Algorithm for Frequency Relaying using FPGAs," Proc. IEEE PES General Meeting, Calgary, pp. 1-6.

Dash, P. K., Pradhan, A. K. and Panda, G. (1999) "Frequency estimation of distorted power system signals using extended complex Kalman filter," IEEE Trans. Power Del., vol. 14, pp. 761-766.

Dash, P. K., Swain, D. P., Routray, A. and Liew, A. C. (1997) "An adaptive neural network approach for the estimation of power system frequency," Electric Power Systems Research, vol. 41, pp. 203-210.

Deb, K. (2002) Multi-objective optimization using evolutionary algorithms, John Wiley \& Sons.

De Jong, K. A. (2006) Evolutyionary compuation: a unified approach, MIT Press.

EEUG (1987), Alternative Transients Program Rule Book. LEC. 
El-Naggar, K. M. and Youssef, H. K. M (2000) "A genetic based algorithm for frequency-relaying applications," Electric Power Systems Research, vol. 55, pp. 173-178.

Goldberg, D. E. (2002) "Design of innovation: lessons from and for competent genetic algorithms", Kluwer Academic Publishers, Norwell, USA.

Karimi, H., Karimi-Ghartemani, M. and Iravani, M. R. (2004) "Estimation of frequency and its rate of change for applications in Power Systems," IEEE Trans. Power Del., vol. 19, pp. 472-480.

Lee, D. C. (1992) Ed., IEEE Recommended Practice for Excitation System Models for Power System Stability Studies (IEEE Std 421.5-1992). Energy Development and Power Generating Committee of the Power Engineering Society.

Mukherjee, V. and Ghoshal, S. (2007) "Intelligent particle swarm optimized fuzzy PID controller for AVR System," Electric Power Systems Research, vol. 77, pp. 1689-1698.

Phadke, A. G., Thorp, J. S. and Adamiak, M. G. (1983) "A new measurement technique for tracking voltage phasors local system frequency, and rate of change of frequency" IEEE Trans. Power App. Syst., vol. PAS-102, pp. 1025-1038.

Routray, A., Pradhan, S. K. and Rao, K. P. (2002) "A novel Kalman filter for frequency estimation of distorted signals in power systems,"IEEE Trans. Instrum. Meas., vol. 51, pp. 469-479.

Sachdev, M. S. and Giray, M. M. (1985) "A least error squares technique for determining power system frequency," IEEE Trans. Power App. Syst., vol. PAS-104, pp. 437-444.

Souza, S. A., Oleskovicz, M., Coury, D. V., Silva, T. V., Delbem, A. C. B. and Simões, E. V. (2008) "FPGA implementation of genetic algorithms for frequency estimation in power systems," in Proc. IEEE PES General Meeting, Pittsburgh, pp. 1-6.

Terzija, V. V., Djuric, M. B. and Kovacevic, B. D. (1994) "Voltage phasor and local system frequency estimation using Newton type algorithm," IEEE Trans. Power Del., vol. 9, pp. 1368-1374.

Vieira Filho, X. (1984) Operation of Power System with Automatic Control. Rio de Janeiro: Editora Campus Ltda.

Wang, M. and Sun, Y. (2004) "A practical, precise method for frequency tracking and phasor estimation," IEEE Trans. Power Del., vol. 19, pp. 1547-1552.
Ziarani, A. K. and Konrad, A. (2004) "A method of extraction of nonstationary sinusoids," Signal Processing, vol. 84, pp. 1323-1346. 